\title{
Evaluation of the anesthetic depth and bispectral index during propofol sequential target-controlled infusion in dogs
}

\author{
Matheus Luis Cunha Ubiali(D), Guilherme Paes Meirelles, Julia Milczewski Vilani(i), Henrique Erick da Luz@D,
} Sabrine Marangoni (D), Raisa Braul Rodrigues $\mathbb{D}$ and Ricardo Guilherme D’OCtaviano de Castro Vilani(D)

\begin{abstract}
Department of Veterinary Sciences Graduation, Agricultural Sciences Sector, Federal University of Paraná, Curitiba, Brazil. Corresponding author: Matheus Luis Cunha Ubiali, e-mail: ubialimatheus@gmail.com

Co-authors: GPM: mv.guilhermepm@gmail.com, JMV: juliavalina@gmail.com, HEL: henriqueerickdaluz@gmail.com, SM: sabrine.marangonivet@gmail.com, RBR: raisabraul@gmail.com, RGDC: ricardovilani@gmail.com,

Received: 22-10-2021, Accepted: 27-01-2022, Published online: 08-03-2022
\end{abstract}

doi: www.doi.org/10.14202/vetworld.2022.537-542 How to cite this article: Ubiali MLC, Meirelles GP, Vilani JM, da Luz HE, Marangoni S, Rodrigues RB, Vilani RG (2022) Evaluation of the anesthetic depth and bispectral index during propofol sequential target-controlled infusion in dogs, Veterinary World, 15(3): 537-542.

\begin{abstract}
Background and Aim: The use of anesthetic infusions based on pharmacokinetic values associated with anesthetic plan and bispectral index in dogs have not been well-documented in the literature. This study aimed to evaluate the bispectral index (BIS) change based on pre-propofol and establish clinical anesthetic depth changes during propofol sequential targetcontrolled infusion (STCI) in dogs with a plasma target of $5 \mu \mathrm{g} / \mathrm{mL}$.
\end{abstract}

Materials and Methods: Twenty healthy male dogs aged 1-3 years and weighing 9.8-44 kg were recruited. These dogs were pre-medicated intramuscularly with methadone $(0.2 \mathrm{mg} / \mathrm{kg})$ and acepromazine $(0.03 \mathrm{mg} / \mathrm{kg})$. After $30 \mathrm{~min}$, propofol anesthetic induction and maintenance were initiated using STCI according to dog pharmacokinetic (PK) parameters. Subsequently, the target plasma concentration of propofol was set at $5 \mu \mathrm{g} / \mathrm{mL}$ for both anesthetic induction and the 120 min maintenance. Then, TivaTrainer v.9.1 software was used to calculate anesthetic infusion rates in a TCI plasmatic concentration mode using the PKs model optimized by covariates for propofol TCI in dogs. The BIS value was recorded every $5 \mathrm{~min}$ from the beginning of induction until the end of anesthesia. Finally, analysis of variance was performed on numerical data using the Friedman test, followed by the Bonferroni adjustment $(\mathrm{p}<0.05)$.

Results: A statistical difference was observed between the baseline BIS value (T0), with a median value of 84.5 (81-97), and BIS after every $15 \mathrm{~min}$ (T15) of inducing anesthesia. Surgical anesthetic depth was also reached in 18 of $20 \mathrm{dogs}$ after $10 \mathrm{~min}$ of infusion and in all dogs after 20 min, with a median BIS value of 72 (53-89) at the time of surgical anesthesia depth. Results also showed no BIS variation $(\mathrm{p}<0.05)$ between anesthetic moments after anesthetic induction with a substantial amplitude of BIS in the surgical anesthetic depth. Moreover, the maximum depth of anesthesia in all dogs by clinical evaluation was reached after 20 min of anesthesia and then remained stable throughout the anesthetic period.

Conclusion: This study suggested that most dogs (90\%) attained a surgical depth of anesthesia within 15 min of STCI onset, with a plasma target of $5 \mu \mathrm{g} / \mathrm{mL}$ and no change in anesthetic depth throughout the period anesthesia lasted. Furthermore, median BIS values remained high even after dogs reached the surgical depth of anesthesia, indicating that the comparison of BIS values of dogs and humans should not be considered for classifying anesthetic and hypnotic depths in dogs.

Keywords: bispectral index, target-controlled infusion, total intravenous infusion.

\section{Introduction}

Propofol is an intravenous anesthetic drug that produces rapid hypnosis, has a short duration of action, and has favorable pharmacokinetic (PK) characteristics for use during continuous infusions. As with most anesthetics, propofol is a gamma-aminobutyric acid (GABA) agonist receptor. In addition, it has a favorable PK and pharmacodynamic (PD) profile, resulting in its widespread use as an intravenous anesthetic [1]. Total intravenous anesthesia (TIVA) involves using injectable drugs intermittently and at constant rate infusions or target-controlled infusion

Copyright: Ubiali, et al. Open Access. This article is distributed under the terms of the Creative Commons Attribution 4.0 International License (http://creativecommons.org/licenses/by/4.0/), which permits unrestricted use, distribution, and reproduction in any medium, provided you give appropriate credit to the original author(s) and the source, provide a link to the Creative Commons license, and indicate if changes were made. The Creative Commons Public Domain Dedication waiver (http://creativecommons.org/ publicdomain/zero/1.0/) applies to the data made available in this article, unless otherwise stated.
(TCI) to provide balanced analgesia, paralysis, and a state of amnesia or unconsciousness. It can also offer a rapid induction and recovery from anesthesia and decrease post-anesthetic nausea and vomiting [2]. Although TIVA is a recent clinical trend, it is not new. The first documented report of TIVA was in the dog in 1656 [3].

TCI is an anesthetic technique that uses species-specific PK models to calculate rates of anesthetic agent delivered by infusion pumps to achieve and maintain a target plasma concentration. The main difference between TCI and conventional infusions is that TCI slows the infusion rate at regular intervals to account for drug absorption into saturable compartments according to the infusion time and PK model used [4].

The absence of commercial TCI pumps for dogs makes this technique unfeasible in clinical practice. However, some TCI software options accepting user-defined PK parameters and driving selected 
syringe pumps are available. Examples include Rug Loop I and computer-controlled infusion pump linked to specific models of syringe pumps. In the absence of particular syringe pumps to use the TCI technique in the clinical setting of the veterinary anesthetic routine, this study also proposed infusion pumps with sequential infusion rate functions. However, this absence does not limit the use of the TCI technique exclusively for specific software and pumps, making it possible to be used in clinical routines.

This study aimed to evaluate the possible correlation between variations in bispectral index (BIS) values and anesthetic depth in dogs anesthetized through a more clinically accessible approach to TCI. The hypothesis investigated in this study was the possibility of using sequential TCI (STCI) with a plasma target of propofol $(5 \mu \mathrm{g} / \mathrm{mL})$ to induce and maintain anesthesia in dogs. In addition, we compared the anesthetic plan with the BIS value.

\section{Materials and Methods}

\section{Ethical approval}

The study was approved by the Ethics Committee of the Agricultural Sciences campus of the Universidade Federal do Paraná (Federal University of Paraná, Brazil), in the session of February 28, 2020 (No. 007/2020).

\section{Study design, period, and location}

This prospective experimental study was performed from July 2020 to May 2021. It was conducted in the Veterinary Hospital UFPR, situated in Curitiba, State of Paraná, Brazil.

\section{Procedures}

Twenty male dogs, classified using ASA I (categorization of patients using the American Society of Anesthesiologists patient status scale scoring of 1-5) [5], with their body masses ranging between $9.8 \mathrm{~kg}$ and $44 \mathrm{~kg}$, were included in the study. Animals were classified as healthy after clinical and laboratory evaluation, after which they were acclimatized to an individual cage $60 \mathrm{~min}$ before starting the anesthetic procedure. Subsequently, all dogs were pre-medicated intramuscularly using a combination of $0.03 \mathrm{mg} / \mathrm{kg}$ acepromazine (Acepran $0.2 \%$, Vetnil, SP, Brazil) and $0.2 \mathrm{mg} / \mathrm{kg}$ methadone (Mytedom 1\%, Cristália, SP, Brazil). Then, anesthesia was induced 30 min later and maintained with propofol (Propotil 1\%, BioChimico, RJ, Brazil) using STCI according to the dog PK parameters proposed by Cattai et al. [6], using the plasma concentration of propofol $(5 \mu \mathrm{g} / \mathrm{mL})$ as a target. Next, PK data, the volume of distribution, and intercompartmental transfer constants used were obtained from the modeling proposed by Cattai et al. [6], considering the following covariates: Weight, age, size, premedication, and sex. PK values were also modeled for each animal and entered the TivaTrainer v.9.1 software (https://www.tivatrainer.com/) using the TCI function plasmatic concentration mode. Afterward, the program generated the individual infusion sequence for each dog's induction and maintenance of plasma concentration. Later, this sequence was manually entered into the infusion pump (HP TCI, Medcaptain, China) at a sequential flow variation mode before induction. Finally, anesthesia was maintained for $120 \mathrm{~min}$.

\section{Infusion rates}

The first infusion rate of propofol calculated using the software was considerably higher than the others. This result represented a $20 \mathrm{~s}$ bolus, raising the blood plasma concentration of propofol from 0 to $5 \mu \mathrm{g} / \mathrm{mL}$. This rate varied between 1.98 and $2.39 \mathrm{mg} / \mathrm{kg} / \mathrm{min}$, representing an initial dose range between 0.66 and $0.8 \mathrm{mg} / \mathrm{kg}$, respectively. After $20 \mathrm{~s}$ of infusion, the rate dropped to values between 0.59 and $0.73 \mathrm{mg} / \mathrm{kg} / \mathrm{mg}$, representing a reduction of approximately $70 \%$ compared with the initial rate. However, after $40 \mathrm{~s}$ of infusion, the infusion rate dropped from $1 \%$ to $5 \%$ every $20 \mathrm{~s}$ in the first $8 \mathrm{~min}$. After $8 \mathrm{~min}$ from the start of the infusion, the maximum decrease in infusion rate was 3\% every 4 min until 40 min of anesthesia. After $40 \mathrm{~min}$ from the start of the infusion, the infusion rate remained practically stable from then on (between 0.2 and $0.37 \mathrm{mg} / \mathrm{kg} / \mathrm{min}$ ) for each $\mathrm{dog}$, but with a continuous rate of decrease, less than or equal to $1 \%$, every 4 min until the end of the anesthesia period.

\section{BIS measurement}

The value of BIS was assessed using BIS Vista 3.01 (Aspect Medical Systems Inc., Mansfield, MA02048, USA). First, electrodes (Covidien, Mansfield, MA 02048, USA) were positioned as described by Guerrero and Nunes [7]. Then, to ensure strong brainwave signals, signal quality index and electromyogram values were set at $>30$. Subsequently, BIS values were recorded immediately before the start of propofol administration (T0) and every $5 \mathrm{~min}$ (T5, T10 to T120) until the end of the anesthesia (T120).

\section{Anesthetics parameters and monitoring}

Anesthetic depth was assessed clinically following the modified classification described by Bleijenberg et al. [8]. Specifically, superficial anesthesia (palpebral and corneal reflexes present; poor muscle relaxation; and mandibular tone should be present), surgical anesthesia (palpebral reflexes sluggish or absent; jaw muscle tone relaxed; and rostroventral rotation of eyeballs), or deep anesthesia (profound muscle relaxation and eye centrally fixed) were used. After losing the cough reflex, dogs were intubated and mechanically ventilated.

Subsequently, after orotracheal intubation with an appropriately sized tube for each dog, controlled intermittent positive pressure ventilation with $100 \%$ inspired oxygen fraction was instituted throughout anesthesia for all dogs. Next, the ventilator (VentPet Plus, RZVET, Brazil) was initially configured with an inspiratory: expiratory (I: E) ratio of 1:2, a respiratory rate (fR) (10 movements/min), and peak pressure $\left(10 \mathrm{~cm} \mathrm{H}_{2} \mathrm{O}\right)$. The $\mathrm{fR}$ and peak pressure values were 
also varied to maintain the end-tidal carbon dioxide pressure between 35 and $45 \mathrm{~mm} \mathrm{Hg}$.

In addition, a convection heating system (WarmAir WA7001, Gentherm Medical, USA) was configured to maintain the esophageal temperature between $36^{\circ} \mathrm{C}$ and $38^{\circ} \mathrm{C}$ throughout the procedure. Then, heart rate and rhythm were measured by electrocardiogram monitor (TEB-ECGPC VET, Brazil) using a bipolar II lead. After starting anesthesia, direct systolic blood pressures and peripheral tissue oxygen saturation were recorded using a multiparametric monitor (RM1000vet, RZVET). After $60 \mathrm{~min}$ of beginning the anesthetic induction, lidocaine (Hypocaína, $20 \mathrm{mg} / \mathrm{kg}$, Hypofarma, MG, Brazil) was injected at the rate of $1.5 \mathrm{mg} / \mathrm{kg}$ per application point in intratesticular and pre-scrotal skin incision line. Finally, the surgical procedure (bilateral orchiectomy) started 20 min after executing local blocks. The maximum and minimum times of the surgical procedure were 10 and $20 \mathrm{~min}$, respectively.

\section{Statistical analysis}

Analysis of variance was performed on numerical data using the Friedman test, followed by the Bonferroni adjustment $(\mathrm{p}<0.05)$. Then, BIS values were grouped according to time since induction. Furthermore, variations in BIS between anesthetic periods were verified using Friedman's non-parametric test, followed by post hoc with the Wilcoxon test and Bonferroni adjustment at $\mathrm{p}<0.05$. Finally, statistical analyses were generated with the $\mathrm{R}$ software Version 4.1.0 (https://www.r-project.org/), using dplyr, rstatix, reshep, PMCMRplus, and ggplot2 packages. Finally, BIS recordings were made 25 times in each animal, totaling 500 measurements.

\section{Results}

A statistically significant difference was observed between the baseline BIS value (T0) and all other measurements made more than $15 \mathrm{~min}$ from the start of the infusion. The median baseline BIS value (T0) after the pre-anesthetic medication was 84.5 (81-97). Furthermore, no statistical difference was observed between BIS measurements at $\mathrm{T} 0$ and $\mathrm{T} 5$, median of 83.5 (73-96, $\mathrm{p}=1)$, T10 with a median of $75.5(60-$ 93, $\mathrm{p}=0.342)$, and T15 with a median of 73 (45-89, $\mathrm{p}=0.07$ ) (Figure-1).

Subsequently, the depth of anesthesia was recorded. At T5, the number of dogs at the superficial and surgical anesthesia depths was, respectively, 11 and 9. Although 2 and 18 were recorded at T10, 1 and 19 were recorded at T15. From T20 till the end of anesthesia, all dogs maintained a surgical anesthetic plane with a median BIS value of 72 (53-89). Nevertheless, no dog was recorded in a deep anesthetic plane at any time.

Figure-1 is a representation of boxplot of the BIS values for the 20 dogs measured at 5 min intervals throughout anesthesia. Statistical differences from T0 by the Friedman test with Bonferroni adjustment $(\mathrm{p}<0.05)$ are marked with asterisk $(*)$.

\section{Discussion}

STCI uses TCI simulators in computer programs or a mobile app to calculate a protocol for individual patients. Subsequently, this protocol can be preloaded into an infusion pump in the sequential mode function (or manually administered during anesthesia using a conventional syringe pump). However, new simulations and protocols must be created in real-time when varying the desired target concentrations during anesthesia becomes necessary. If the target plasma concentration is maintained, the anesthetic depth should remain stable throughout the procedure. Nevertheless, anesthetic protocols are designed to maintain hemodynamic stability with an appropriate anesthetic depth in modern anesthesia. Therefore, it can be challenging to measure anesthetic depth [8] since subjective evaluations rely on the anesthetist's experience. Classification of the anesthetic plane has been described since the $19^{\text {th }}$ century when Arthur Guedel characterized the stages of anesthesia and anesthetic depth according to the degree of hypnosis with ether [9].

Similarly, assessing the anesthetic depth in veterinary practice is achieved by monitoring a combination of physiological variables. Yet, the specific physiological variable evaluated in each case can vary depending on the PK and PD properties of the anesthetic drug used. Hence, muscle relaxation, loss of mandibular tone, and assessment of somatic reflexes in the cranial nerves are also used to assess anesthetic depth. Conversely, it has been reported that cranial nerve reflexes are derived from subcortical and spinal structures and can only provide crude estimations of the degree of consciousness [10]. Moreover, other physiological variables used for monitoring autonomic responses, such as blood pressure and heart and respiratory rates, depend highly on the protocol used.

Therefore, combining the BIS to assist the chosen target in the TCI technique in humans showed reduced mean propofol anesthetic maintenance rates than the non-BIS-assisted TCI technique [11]. Compared with our study, some limitations were observed, the main one being the absence of a control group to link and verify the benefit of BIS associated with the TCI technique in this specific case with STCI. Another correlation between BIS values in dogs using the modified Glasgow Coma Scale score with altered levels of consciousness showed the efficiency of BIS in monitoring consciousness in patients with altered levels of consciousness [12]. However, studies directed to the applicability of BIS in dogs are scarce.

The evaluation of the electrical activity of the cerebral cortex, using measurable and objective variables, has also been used to categorize anesthetic depth and guide the titration of general anesthesia [13]. Besides, BIS measures sub-parameters of brain electrical activity, using human algorithms to analyze wave frequencies during electroencephalographic tracing [14]. This index is measured on 


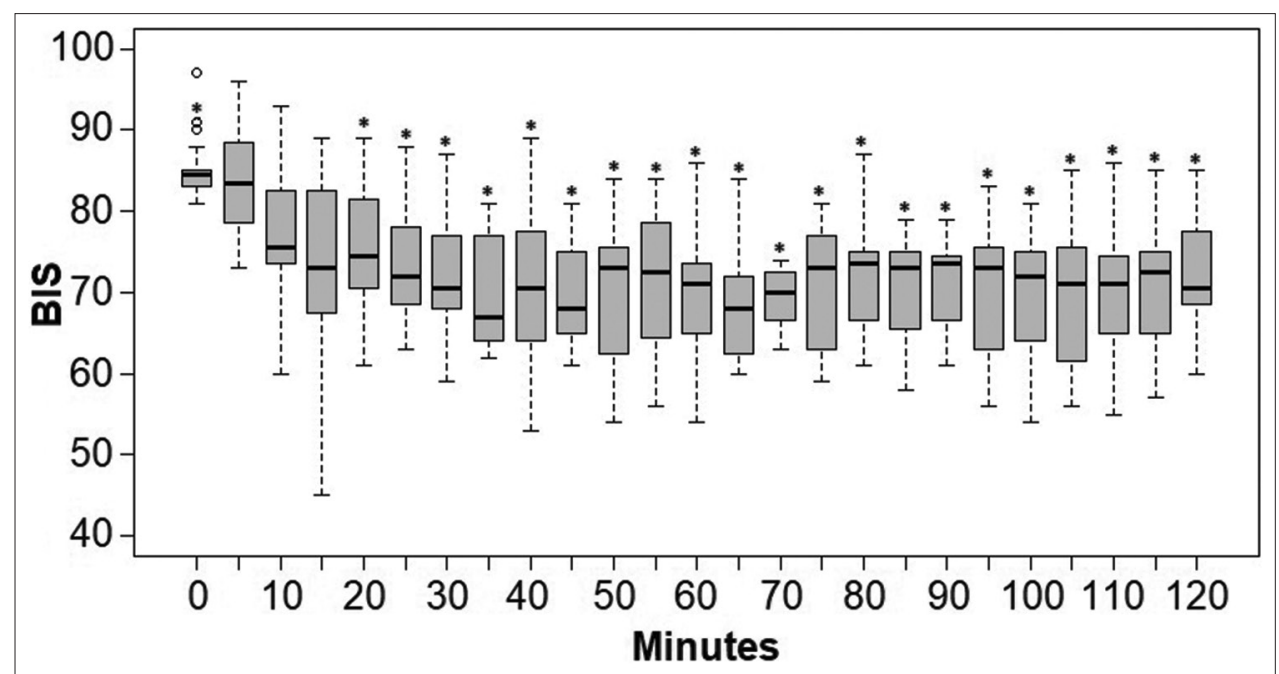

Figure-1: Representation of boxplot of the bispectral index values for the 20 dogs measured at 5 min intervals throughout anesthesia.

a dimensionless unit scale from 0 to 100 [15]. The lower the BIS value, the greater the degree of hypnosis. In humans, although the numerical classification of BIS varies according to the protocol used, it can be used to categorize the anesthetic depth from awake patients through deep sedation and anesthesia to deep hypnosis or isoelectric encephalographic activity [16]. In the study described by Eizadi-Mood et al. [15], the BIS value in humans can be used as a predictor for intubation in unconscious patients, showing a sensitivity of $88 \%$ and specificity of $87 \%$ for endotracheal intubation, with mean BIS values of 66.47 and 85.21 for patients who needed endotracheal intubation and did not need intubation, respectively. Alternatively, in our study with dogs, median BIS values at the time of intubation were higher. Furthermore, the correlation between results obtained in our study of BIS values and moments of intubation in dogs was not verified due to the high amplitude and higher median BIS values observed in dogs during the intubation. Nevertheless, BIS values, appearing higher in dogs than humans, and categorization according to anesthetic depths have not been established [17].

A difference was observed between BIS values, the above expectations, and results observed in our study compared to reference values in human anesthesia surgical depth [18]. This difference was related to the possible limitations associated with neurophysiologic and anatomic differences between dogs and humans, in addition to the difference in available updates of the proprietary BIS algorithm [19]. However, when comparing BIS values in dogs were demonstrated in the previous studies $[8,19]$, we verified that median BIS values obtained in our study were higher than the previous studies. This difference is proposed to be related to the different anesthetic protocols used. Therefore, the PKs and PDs of drugs and their interactions, including the doses and dosages used in pre-anesthetic medication, anesthetic induction, and maintenance, are proposed to justify this difference.

Burst suppression on electroencephalogram (EEG) is defined as suppression periods longer than $0.5 \mathrm{~s}$ during which the amplitude does not exceed $5 \mu \mathrm{V}$ in humans [20]. The BIS version can contribute to the observed differences in BIS values between the previous studies $[8,19]$ and our study. As observed, high BIS values $(>60)$ were associated with EEG burst suppression patterns [21], suggesting that the algorithm was insufficient to calculate BIS in the presence of isoelectric period or hypothermia [22]. This finding indicated that even though the BIS v.3.01 contained an improved EEG burst suppression detection [23], the algorithm used to calculate the BIS at v.3.01 had some problems during processing of the human EEG in patients with hypothermia, especially at burst and suppression EEG [21]. Among hypnosis monitoring devices, EEG is the most common in detecting electrical activity of the cortex [24]. The last version available for upload in early 2021 was the BIS 3.5 version. Unfortunately, this version is only available on 4-channel BIS monitors.

The reduction in BIS is related to the amount of propofol in the central nervous system (CNS) and hence to the degree of CNS hypnosis. One of the factors determining the quantity of propofol in the CNS is the propofol transfer rate from the blood tissue to the CNS. It depends on several factors, such as the cardiac output, cerebral blood flow, and drug PK properties that determine the transfer rate across the blood-brain barrier (liposolubility and degree of drug ionization, integrity of tissue membranes, and difference in drug concentrations between compartments).

The statistical difference between the observation moments of the BIS value is proposed to be correlated with the chosen target value. For example, higher targets will have higher infusion rates than low targets, consequently increasing the effect of propofol on the CNS and changing the BIS value. Another 
essential factor correlated with variations in the BIS value is the type of software update used in the BIS monitors. Updates before version 3.01 are susceptible to overestimation of the BIS value in moments of EEG burst suppression detection.

Therefore, the BIS monitor has been made available for more than 20 years for human clinical use and has had an immense impact on academic activity in anesthesiology [25]. However, dog-specific BIS development is required, including a solid and vibrant theoretical basis of the methodology used in forming the sub-parameters used in the EEG algorithms to generate the final BIS value. These considerations would reduce possible failures and errors in using human BIS algorithms for dogs.

\section{Conclusion}

This study suggested that it is possible to use the TCI technique in dogs with anesthetic depth stability throughout anesthesia, even in the absence of specific pumps during the clinical routine. However, the applicability of STCI in the clinical routine should be further studied since some animals are proposed to require more time to reach the surgical depth of anesthesia and thus the use of different plasma targets. Furthermore, BIS values remained constant and in high median values even after dogs attained the surgical depth of anesthesia, demonstrating that comparing BIS values with those in humans should not be considered for classifying anesthetic and hypnotic depths in dogs.

\section{Authors' Contributions}

RGDC and MLCU: Designed the study. MLCU, JMV, RGDC, GPM, HEL, SM, and RBR: Collected the samples, performed the experiments, and contributed to the original draft. MLCU and RGDC: Review of the manuscript and contributed to the editing of the manuscript. All authors read and approved the final manuscript.

\section{Acknowledgments}

The authors are grateful to the Department of Agricultural Sciences Sector, Federal University of Paraná, Curitiba, Brazil, for providing the necessary facilities for this study. The authors did not receive any funds for this study.

\section{Competing Interests} interests.

The authors declare that they have no competing

\section{Publisher's Note}

Veterinary World remains neutral with regard to jurisdictional claims in published institutional affiliation.

\section{References}

1. Sahinovic, M.M., Struys, M.R.F. and Absalom, A.R. (2018) Clinical pharmacokinetics and pharmacodynamics of propofol. Clin. Pharmacokinet., 57(2): 1539-1558.

2. Raffe, M.R. (2020) Total intravenous anesthesia for the small animal critical patient. Vet. Clin. North Am. Small Anim. Pract., 50(6): 1433-1444.

3. Chong, C.T. (2018) Historical perspectives on total intravenous anaesthesia (TIVA). J. Anesth. Hist., 4(1): 60.

4. Schnider, T.W., Minto, C.F., Struys, M.M.R. and Absalom, A.R. (2016) The safety of target-controlled infusions. Anesth. Anal., 122(1): 79-85.

5. Grubb, T., Sager, J., Gaynor, J.S., Montgomery, E., Parker, J.A., Shafford, H. and Tearney, C. (2020) 2020 AAHA anesthesia and monitoring guidelines for dogs and cats. J. Am. Anim. Hosp. Assoc., 56(2): 59-82.

6. Cattai, A., Bizzotto, R., Cagnardi, P., Cesre F.D. and Franci, P. (2019) Pharmacokinetic model optimized by covariates for propofol target-controlled infusion in dogs. Vet. Anaesth. Anal., 46(5): 568-578.

7. Guerrero, P.N.H. and Nunes, N. (2003) Monitoramento do índice bispectral em cães. Semi. Ciên. Agrár. Londrina., 24(1): 163-170.

8. Bleijenberg, H.E., Oostrom, H., Akkerdaas, L.C., Doornenbal, A. and Hellebrekers, L.J. (2011) Bispectral index and the clinically evaluated anaesthetic depth in dogs. Vet. Anaesth. Analg., 38(6): 536-543.

9. Siddiqui, B.A. and Kim, P.Y. (2021) Anesthesia Stages. StatPearls. Available from: https://www.ncbi.nlm.nih.gov/ books/NBK557596. Retrieved on 21-10-2021.

10. March, P.A. and Muir, W.W. (2005) Bispectral analysis of the electroencephalogram: A review of its development and use in anesthesia. Vet. Anaesth. Analg., 32(5): 241-255.

11. Lin, Y.J., Wang, Y.C., Huang, H.H., Huang, C.H., Liao, M.X., and Lin, P.L. (2019) Target-controlled propofol infusion with or without bispectral index monitoring of sedation during advanced gastrointestinal endoscopy. $J$. Gastroenterol. Hepatol., 35(7): 1189-1195.

12. Saenubol, P.P., Akatvipat, A., Pleumsamran, A. and Chankrachang, S. (2021) Correlation between bispectral index value and modified Glasgow Coma Scale score in dogs with altered level of consciousness. J. Vet. Emerg. Crit. Care, 31(1): 52-58.

13. Oliveira, C.R.D., Bernardo, W.M. and Nunes, V.M. (2017) Benefit of general anesthesia monitored by bispectral index compared with monitoring guided only by clinical parameters. Systematic review and meta-analysis. Braz. J. Anesthesiol., 67(1): 72-84.

14. Mathur, S., Patel, J., Goldstein, S. and Jain, A. (2021) Bispectral index. StatPearls. Available from: https:// www.ncbi.nlm.nih.gov/books/NBK539809 Retrieved on 10-21-2021.

15. Eizadi-Mood, N., Halakoei, L., Yaraghi, A., Jabalameli, M., Talakoub, R. and Sabzghabaee, A.M. (2018) Bispectral index in poisoning cases with multi-drug ingestion: A predictable role for early endotracheal intubation. J. Res. Pharm. Pract., 7(1): 36-40.

16. Bard, J.W. (2001) The BIS monitor: A review and technology assessment. AANA J., 69(6): 477-483.

17. Lopes, P.C.F., Nunes, N., Paula, D.P., Nishimori, C.T.D., Guerrero, P.N.H. and Conceição, E.D.V. (2008) Bispectral index in dogs at three intravenous infusion rates of propofol. Vet. Anaesth. Analg., 35(3): 228-231.

18. Kim, D., Ahn, J.H., Heo,G. and Jeong, J.S. (2021) Comparison of bispectral index and patient state index values according to recovery from moderate neuromuscular block under steady-state total intravenous anesthesia. Sci. Rep., 11(1): 5908.

19. Campagnol, D., Neto, F.J.T., Monteiro, E.R., Beier, S.L. and Aguiar, A.J.A. (2007) Use of bispectral index to monitor depth of anesthesia in isoflurane-anesthetized dogs. $\mathrm{Am}$. J. Vet. Res., 68(12): 1300-1307.

20. Koyama, C., Haruna, T., Hagihira, S. and Yamashita, K. (2019) New criteria of burst suppression on electroencephalogram in dogs anesthetized with sevoflurane. Res. Vet. Sci., 


\section{3(1): 171-177.}

21. Detsch, O., Schneider, G., Kochs, E. and Werner, C. (2000) Increasing isoflurane concentration may cause paradoxical increases in the EEG bispectral index in surgical patients. Br. J. Anaesth., 84(1): 33-37.

22. Doi, M., Gajraj, R.J., Mantzaridis, H. and Kenny, G.N. (1997) Effects of cardiopulmonary bypass and hypothermia on electroencephalographic variables. Anaesthesia, 52(11):
1048-1055.

23. Johansen, J.W. and Sebel, P.S. (2000) Development and clinical application of electroencephalographic bispectrum monitoring. Anesthesiology, 93(5): 1336-1344.

24. Garra, R. and Riso, C. (2020) Monitoring anesthesia depth: Look to the brain. Minerva Anestesiol., 86(6): 586-588.

25. Connor, C.W. (2020) A forensic disassembly of the BIS monitor. Anesth. Analg., 131(6): 1923-1933.

$* * * * * * * *$ 